\title{
Towards Mapping Perovskite Oxide 3-D Structure Using Two-Dimensional Pixelated STEM Detector
}

\author{
Magnus Nord ${ }^{1}$, Andrew Ross ${ }^{1}$, Ingrid Hallsteinsen², Thomas Tybell ${ }^{2}$, Ian MacLaren ${ }^{\mathbf{1}}$ \\ 1. School of Physics and Astronomy, University of Glasgow, Glasgow, United Kingdom \\ 2. Department of Electronics and Telecommunication, Norwegian University of Science and Technology, \\ Trondheim, Norway.
}

Perovskite materials can exhibit a wide range of properties like superconductivity, magnetism and piezoelectricity. These diverse functional properties emerge due to strong structure - function coupling. Small changes in lattice structure can lead to large changes in functional properties. In particular, perovskite heterostructures have recently attracted much interest due to the possibility of tailoring properties through coupling between substrate and film. However, due to the many different parameters that can affect the properties of these materials, one needs detailed information about the structure of the films, especially at the film/substrate interface. In particular, the promotion or suppression of octahedral tilt ordering at interfaces, and the measurement of this using scanning transmission electron microscopy (STEM) is of great interest [1-3]. Ideally one wants to have precise information about every atom position to get the full 3-D structure. Using STEM one can normally get high resolution structural information about the axes orthogonal to the electron beam, however this still leaves one axis unaccounted for. Normally, two different TEM-lamellae thinned on two orthogonal zone axes is required to get this information about all three axes. However, extracting all this from one region of one sample would obviously be advantageous.

Recently, advances in electron counting devices have enabled the development of fast 2-D pixelated STEM detectors. Such detectors can acquire a diffraction pattern for each probe position in a STEM scan, yielding a 4-D dataset: two spatial dimensions in the sample plane and two reciprocal dimensions in the detector plane. This allows for imaging of large parts of the convergent beam electron diffraction pattern (CBED) generated when doing STEM. This diffraction pattern contains a wealth of information, including the Higher Order Laue Zones (HOLZ), which reveals information about the crystallographic ordering parallel to the electron beam [4].

In this work, we have used a probe corrected ARM200cF equipped with a Medipix3 pixelated STEM detector to acquire CBED datasets across a $\mathrm{La}_{0.7} \mathrm{Sr}_{0.3} \mathrm{MnO}_{3} / \mathrm{LaFeO}_{3}(\mathrm{LSMO} / \mathrm{LFO})$ heterostructure grown epitaxially on a $\mathrm{SrTiO}_{3}-(111)$ substrate. By radially integrating these diffraction patterns, the datasets are analysed using a model-based approach similar to that previously used in EELS processing [5]. By fitting a Gaussian function to the integrated HOLZ circle (shown in Fig. 1c), information about the lattice size parallel to the electron beam is extracted for both the films and substrate: the HOLZ ring chosen corresponds to a period doubling of the perovskite lattice along the beam direction. By correlating this data to standard atomically resolved STEM images, a more complete picture of the 3-D structure could be obtained. Results from the model-based data processing can be seen in Fig. 1(e-f), which show the integrated intensity of the Gaussian fitted to the HOLZ ring. This shows clearly that this HOLZ ring increases gradually in intensity over several unit cells in the LFO before reaching a maximum, and then falling again in intensity towards and into the LSMO layer. The obvious reason for the appearance of a HOLZ ring corresponding to unit cell doubling in a perovskite would be a octahedral tilting transition, and it is known that bulk LFO has an orthorhombic structure with a doubled 
unit cell along two of the primitive $<110>$ directions resulting from strong octahedral tilting. In contrast to this, $\mathrm{SrTiO}_{3}$ has a primitive cubic perovskite structure, and LSMO of this composition has octahedral tilting of a much reduced magnitude. This correlates almost exactly with the plot seen in Fig. 1f, and we postulate that the intensity in the HOLZ ring will correlate well with the degree of ordering due to octahedral tilting and therefore mapping this intensity using scanned diffraction provides a rapid method for the mapping of octahedral tilting along the beam direction. This could be correlated with the in-plane ordering of octahedral tilting, which can already be determined from atomically resolved STEM imaging, but with the additional advantage that it will not suffer from any displacements of oxygen column images by extraneous imaging effects [6][7].

[1] A. Y. Borisevich et al, Physical Review Letters 105 (2010), p. 087204.

[2] Young-Min Kim et al, Advanced Materials 25 (2013), p. 2497.

[3] Qian He et al, ACS Nano 9 (2015), p. 8412.

[4] F.-T Huang et al, Physical Review Letters 105 (2010), p. 125502.

[5] J. Verbeeck and S. Van Aert, Ultramicroscopy 101 (2004), p. 207.

[6] Dan Zhou et al, Ultramicroscopy 160 (2016), p. 110.

[7] The authors are indebted to the EPSRC for the funding of this work via the project "Fast Pixel

Detectors: a paradigm shift in STEM imaging” (Grant reference EP/M009963/1)
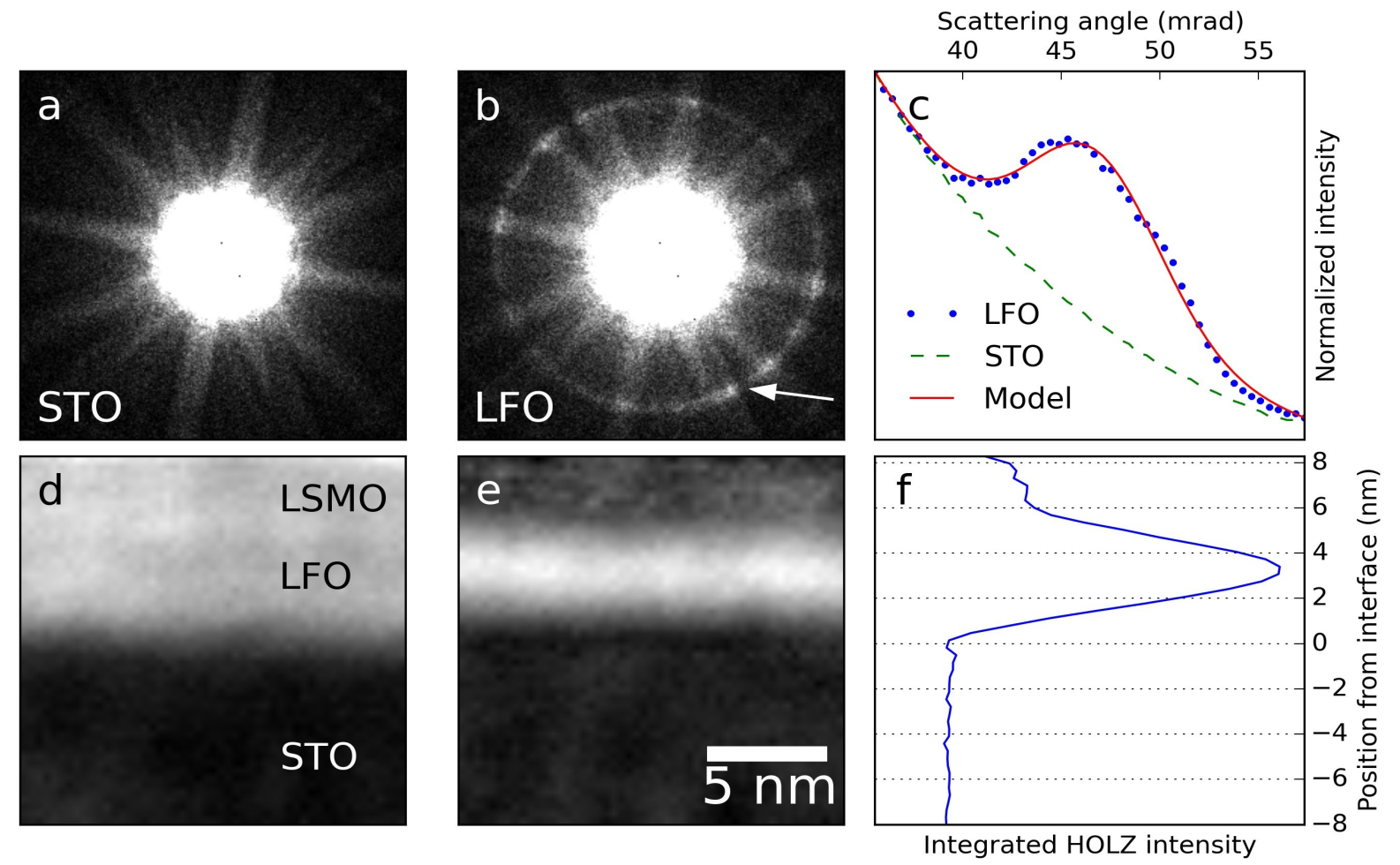

Figure 1. Data processing using 4-D STEM diffraction to extract HOLZ information. (a) STEM diffraction image from STO. (b) Same as (a) but from LFO. Arrow highlighting the HOLZ circle, which is due to unit cell doubling in LFO. (c) Radial integration of the patterns in (a) and (b), and modeling of the HOLZ using a power law for the background and a Gaussian function for the HOLZ. (d) LAADF image constructed from the 4-D dataset by using a virtual aperture. (e) Integrated intensity of the Gaussian used in modeling the HOLZ circle as seen in (c), but for every probe position. Shows the intensity of the HOLZ, which is highest in the middle of the LFO film. (f) Summed version of (e), showing the HOLZ intensity as a function of distance from the LFO/STO interface. 